\title{
CT-measured pulmonary artery diameter as an independent predictor of pulmonary hypertension in cystic fibrosis
}

\author{
Mehrdad Bakhshayeshkaram ${ }^{1 A, D, E}$, Farahnaz Aghahosseini ${ }^{1 A, B, C, E, E, F}$, Fatemeh Vaezi ${ }^{1 B}$, Shahram Kahkooei ${ }^{10, E}$, \\ Yalda Salehi ${ }^{2 E}$, Maryam Hassanzad ${ }^{1 A, B, C D, E, F}$, Hamid Reza Jamaati ${ }^{30, E, F}$, Ali Akbar Velayati ${ }^{4 A, D, F}$ \\ 'Pediatric Respiratory Diseases Research Center, National Research Institute of Tuberculosis and Lung Diseases (NRITLD), Shahid Beheshti University \\ of Medical Sciences, Tehran, Iran \\ ${ }^{2}$ Imam Khomeini Hospital, Tehran University of Medical Science, Tehran, Iran \\ ${ }^{3}$ Chronic Respiratory Diseases Research Center, National Research Institute of Tuberculosis and Lung Diseases (NRITLD), Shahid Beheshti University \\ of Medical Sciences, Tehran, Iran \\ ${ }^{4}$ Mycobacteriology Research Center (MRC), National Research Institute of Tuberculosis and Lung Diseases (NRITLD), Shahid Beheshti University \\ of Medical Sciences, Tehran, Iran
}

\section{Abstract}

Purpose: The role of computed tomography (CT) scan, as a promising prognostic imaging modality in cystic fibrosis (CF), has been widely investigated, focusing on parenchymal abnormalities. The aim of the present study was to evaluate the diagnostic performance of thoracic vascular parameters on CT to detect pulmonary hypertension $(\mathrm{PH})$.

Material and methods: CF patients who contemporaneously underwent CT and echocardiography were retrospectively enrolled. Baseline characteristics in addition to pulmonary artery diameter (PAD) and pulmonary to aortic (PA/A) ratio were compared between cohorts with and without $\mathrm{PH}$, based on the results of echocardiography separately in paediatric patients $(<18)$ and adults $(\geq 18)$.

Results: Of a total 119 CF patients, 39 (32.8\%) had PH (paediatric: 23/78, 29.5\%, adult: $16 / 41,39 \%$ ). In paediatric CF patients, mean age, $\mathrm{HCo}_{3}, \mathrm{PCo}_{2}$, and pulmonary artery diameter (PAD) were significantly higher in the $\mathrm{PH}$ group compared to the non-PH group. Mean pulmo however, only PAD remained as the independent predictor of $\mathrm{PH}$ based on multivariate analysis (overall: $22.86 \mathrm{~mm}[ \pm 3.86]$ vs. $18.43 \mathrm{~mm}[ \pm 4.72], p=0.005$, paediatric patients: $22.63 \mathrm{~mm}$ [ \pm 4.4$]$ vs. $17.10 \mathrm{~mm}[ \pm 4.64], p=0.03)$. Using a cut off of $19.25 \mathrm{~mm}$, the diagnostic performance of PAD to detect PH was found to be as follows: sensitivity $=82 \%$, specificity $=70 \%$, and accuracy $=73.1 \%$. No significant difference was demonstrated in PAD between PH and non-PH groups in adults with CF (23.19 [ \pm 3.60$]$ vs. $21.34[ \pm 3.49], p=0.7)$.

Conclusions: In CF patients, PAD revealed an age-dependent performance to detect PH. PAD can be applied to predict pulmonary hypertension in paediatric CF patients and may be recommended to be routinely measured on follow-up chest CT scan in childhood CF.

Key words: cystic fibrosis, pulmonary hypertension, CT scan, pulmonary artery diameter, pulmonary artery diameter in CF pulmonary pressure.

\section{Introduction}

Pulmonary hypertension (PH) is commonly encountered in advanced cystic fibrosis (CF) and is associated with a poor prognosis. The results of a recent study demon- strated that, regardless of the severity, the hazard ratio of pulmonary hypertension and related impact on mortality is significantly higher in CF patients than that of other lung diseases [1]. Prompt in to a multidisciplinary therapeutic approach, including drug treatment, nutritional

\section{Correspondence address:}

Assoc. Prof. Maryam Hassanzad, Pediatric Respiratory Diseases Research Center, National Research Institute of Tuberculosis and Lung Diseases (NRITLD),

Massih Daneshvari Hospital Daar-Abad, Niavaran Ave., Tehran, Iran, zip code: 19569-44413, post box: 19575-154, phone: 021-27122712,

e-mail: Mar_hassanzad@yahoo.com

Authors' contribution:

A Study design · B Data collection · C Statistical analysis · D Data interpretation - E Manuscript preparation · F Literature search · G Funds collection 
support and respiratory rehabilitation, early detection of $\mathrm{PH}$ in CF patients may hence contribute to prevent or slow disease progression, improving quality of life and probably survival. Echocardiography, as the most sensitive non-invasive screening tool for $\mathrm{PH}$, may not perform as a reliable tool in advanced lung parenchymal diseases, which frequently occurred in CF patients $[2,3]$. In addition, pulmonary function test may not correlate well with the course and severity of pulmonary parenchymal and vascular disease $[4,5]$. Right-sided heart catheterisation as the gold standard for diagnosis of $\mathrm{PH}$ with the capability of providing additional valuable information for patient stratification and treatment strategies is not frequently indicated in CF patients and may be associated with some major complications [6]. There is an increasing body of evidence suggesting a potential role for CT-measured vascular parameters to identify pulmonary hypertension. The results of a recent meta-analysis on 2134 cases from 20 publications revealed that pulmonary artery diameter and pulmonary-to-aorta ratio had a modest sensitivity (79\%) and specificity (81\%) with a good overall accuracy to detect pulmonary hypertension and may play a complementary role in the diagnostic approach to $\mathrm{PH}$ [7]. Providing details about both vascular and parenchymal components, CT may be considered as the imaging modality of choice for disease monitoring in progressive lung diseases; however, evidence about the diagnostic and prognostic implication of CT vascular metric in CF patients is scarce. A recent study revealed that the $\mathrm{PA} / \mathrm{A}$ ratio has a strong correlation with acute respiratory exacerbation and also with cystic fibrosis transmembrane conductance regulator (CFTR) dysfunction in adults with CF and hence may provide valuable prognostic information in this cohort [8]. In addition, few studies are available investigating normal values of thoracic vasculature in the paediatric population [9] as well as any relation between vascular parameters and $\mathrm{PH}$. The aim of the present study was to investigate the relationship between chest CT vascular parameters and pulmonary artery systolic pressure in $\mathrm{CF}$ patients, as well as their performance characteristics to detect $\mathrm{PH}$, separately in paediatric and adult groups.

\section{Material and methods}

\section{Patients}

In this retrospective chart review study, all patients with proven diagnosis of $\mathrm{CF}$ admitted to the paediatric wards of Masih Daneshvari Hospital between 2012 and 2016 were consecutively included. Patients contemporaneously investigated by CT scan and echocardiography with a maximum interval of two weeks were then selected. Cardiopulmonary co-morbidity and $\mathrm{PH}$ with aetiologies other than $\mathrm{CF}$ and aortic aneurysm/dilatation were considered as exclusion criteria. The study was approved by the Institutional Review Board, and the need for informed consent was waived. Demographic and anthropometric characteristics including age, sex, body mass index (BMI), and arterial blood gas indices including $\mathrm{pH}, \mathrm{PCO}_{2}, \mathrm{HCO}_{3}$, pulmonary function test including $\mathrm{FEV}_{1}$ (forced expiratory volume in 1 second; predicted), $\mathrm{FEV}_{1} / \mathrm{FVC}$ (forced vital capacity), and pulmonary artery systolic pressure based on echocardiography were extract from the patients' files and recorded separately for each case. No PFT records were available for patients under the age of six years. Based on pulmonary artery systolic pressure (PASP), patients were then divided into two groups: 1) $\mathrm{PH}$ group, defined as patients with PASP $\geq 25 \mathrm{mmHg}$ and 2) non-PH group, defined as patients with PASP $<25 \mathrm{mmHg}$. Regarding the potential effect of age on the pulmonary artery diameter [10], the study cohort were subsequently categorised into paediatric ( $\leq 18$ years old) and adult CF ( $>18$ years) groups, and all the parameters were then compared between the $\mathrm{PH}$ and non-PH group in each age group, using the same cut-off.

\section{CT acquisition}

CT scan was performed using a spiral CT unit (Siemens SOMATOM Emotion Series, Siemens Erlangen, Germany). All patients were imaged in the supine position, in inspiratory phases from lung apex to below the costophrenic angles, according to the standard acquisition protocol in adults and paediatric patients, as appropriate: $80-100 \mathrm{~mA}$, $110 \mathrm{kVp}, 1-1.4$ pitch, and 0.5-second rotation time. CT scans were reconstructed at section widths of $1 \mathrm{~mm}$ and intervals of $20 \mathrm{~mm}$.

\section{CT vascular metrics}

The widest short-axis diameter of the main pulmonary artery was measured on the axial section at the level of the main pulmonary artery bifurcation in mediastinal window setting (window width 400, window centre 30 ) on expiratory phase images. The widest short-axis diameter of ascending aorta was then measured on an axial section at the same level. The main pulmonary artery diameter (PA) to ascending aorta (A) (PA/A) ratio was then calculated. The measures were performed blinded to the haemodynamic results.

\section{Echocardiography}

Colour Doppler echocardiography was performed using Vivid 7 dimension; Mann healthcare, GE. PASP was calculated as the sum of the peak trans-tricuspid systolic pressure gradient and right atrium pressure, using modified Bernoulli equation. PASP $\geq 25 \mathrm{mmHg}$ was considered as $\mathrm{PH}$.

\section{Statistical analysis}

Normally distributed data were analysed using parametric tests. Continuous (age, $\mathrm{BMI}, \mathrm{HCO}_{3}, \mathrm{PCO}_{2}, \mathrm{FEV}_{1}, \mathrm{FEV}_{1} / \mathrm{FVC}$, 
PASP, PAD, PA/A ratio) and categorical (gender) variables were described as mean $( \pm S D)$ and frequency (percent), respectively. The significance of difference for each variable between two groups was tested using independent $t$-test, Mann-Whitney $U$ test, and chi-square, as appropriate. Correlation between vascular and haemodynamic parameters was analysed using Pearson's correlation test. Variables with significant different based on univariate analysis were further analysed in a logistic regression model. Diagnostic performance of pulmonary artery diameter and PA/A ratio and appropriate cut-off were calculated using receiver operating characteristic (ROC) analysis. A $p$ value $<0.05$ was considered as a statistically significant difference. Data were analysed using SPSS ver. 23.

\section{Results}

Of a total 119 patients with CF, 74 (62.2\%) were male and $45(38.7 \%)$ were female $(p=0.008)$, with mean age of $14.87 \pm 6.4$ years (age range: 3 -33 years). Mean age did not demonstrate a significant difference between male and female (14.4 vs. $15.62, p=0.3$ ).

In this study the mean PAD was $19.88 \mathrm{~mm}$. The mean diameter of the pulmonary artery was greater in adults with CF compared to paediatric patients (22.06 vs. 18.73 , $p=0.000)$.

Regarding haemodynamic parameters, mean PASP was measured as $24.39 \mathrm{mmHg}$. Based on a PASP cutoff of $25 \mathrm{mmHg}$, 39 patients (32.8\%) had increased pulmonary hypertension (mean PASP $=32.8 \mathrm{mmHg}$ ), and $80(67.2 \%)$ showed normal pulmonary systolic arterial pressure (mean PASP $=20.05 \mathrm{mmHg}$ ). The frequency of PH and mean PASP did not show a significant difference between paediatric and adults with CF in this study ( $29.5 \%$ vs. $39 \%, p=0.29$ and 24.08 vs. $24.97, p=0.18$ ).

Among demographic and anthropometric characteristics, mean age was significantly higher in the $\mathrm{PH}$ group (16.94 vs. 13.85 years old, $p=0.015$ ). The $\mathrm{PH}$ paediatric group had also a significantly higher mean age compared to the non-PH paediatric group (14.26 vs. 10.44 years old, $p=0.001$ ); however, such a difference was not demonstrated between $\mathrm{PH}$ and non-PH groups in adult CF patients (22 vs. 20.81, $p=0.22$ ).

Among arterial blood gas indices, mean $\mathrm{PCo}_{2}$ and $\mathrm{HCo}_{3}$ were found to have significantly higher values in the PH group compared to the non-PH cohort (47.98 [ \pm 9.03$]$ vs. $44.11[ \pm 11.70], p=0.011$ and $29.89[ \pm 4.06]$ vs. 27.31 $[ \pm 6.27], p=0.003$, respectively). The same results were obtained for paediatric CF patients $(45.83[ \pm 11.43]$ vs. 43.5 $[ \pm 11.76], p=0.03$ and $38.92[ \pm 4.27]$ and $31.42[ \pm 3.14]$, $p=0.003$, respectively). There was a trend toward a higher mean $\mathrm{HCo}_{3}$ in the $\mathrm{CF}$ adult $\mathrm{PH}$ group than in the non$\mathrm{PH}$ cohort, although it was not statistically significant $(p=0.08)$ (Table 1$)$.

There was a borderline statistical significant difference in $\mathrm{FEV}_{1}$ between the $\mathrm{PH}$ and non-PH group $(p=0.064)$.
While no difference was found in pulmonary function test values between the paediatric $\mathrm{PH}$ and non- $\mathrm{PH}$ group, adult $\mathrm{PH}$ patients had a significantly higher value of $\mathrm{FEV}_{1} /$ FVC compared to the non-PH adult cohort ( $p=0.04)$.

Mean pulmonary arterial diameter and pulmonary-to-aortic (PA/A) ratio were significantly greater in the $\mathrm{PH}$ group than in those with normal pulmonary pressure (22.86 vs. $18.42, p=0.000$ and 1.03 vs. 0.95 , $p=0.002)$. In the paediatric group with CF, CT vascular metrics were also significantly higher in the $\mathrm{PH}$ group than in the non-PH group, although such a difference was not demonstrated in adult CF patients. Table 2 summarises the CT-measured vascular parameters, based on a PASP cut-off of $25 \mathrm{mmHg}$, as the discriminator of $\mathrm{PH}$ from non-PH groups.

There was a statistically significant correlation between haemodynamic (PSAP) and vascular parameters (paediatric patients - PAD: $[\mathrm{R}]=0.59, p=0.000$, $\mathrm{PA} / \mathrm{A}$ ratio: $[\mathrm{R}]=0.47, p=0.00$; adults $-\mathrm{PAD}:[\mathrm{R}]=0.51$, $p=0.006$, $\mathrm{PA} / \mathrm{A}$ ratio: $[\mathrm{R}]=0.45, p=0.023)$. In paediatric patients, the correlation between PSAP and age was also significant $([R]=0.49, p=0.000)$. Pulmonary systolic artery pressure demonstrated a weaker inverse correlation with $\mathrm{HCo}_{3}([\mathrm{R}]=-0.43, p=0.003)$ and $\mathrm{PCo}_{2}([\mathrm{R}]=-0.34$, $p=0.029)$ in children.

Figure 1 demonstrates the area under the ROC curve of pulmonary artery diameter as 0.81 (CI: 0.68-0.85, $p=0.000$ ) and for the PA/A ratio as 0.73 (CI: $0.57-0.78$, $p=0.002)$. Using cut-off of $19.25 \mathrm{~mm}$, the sensitivity and

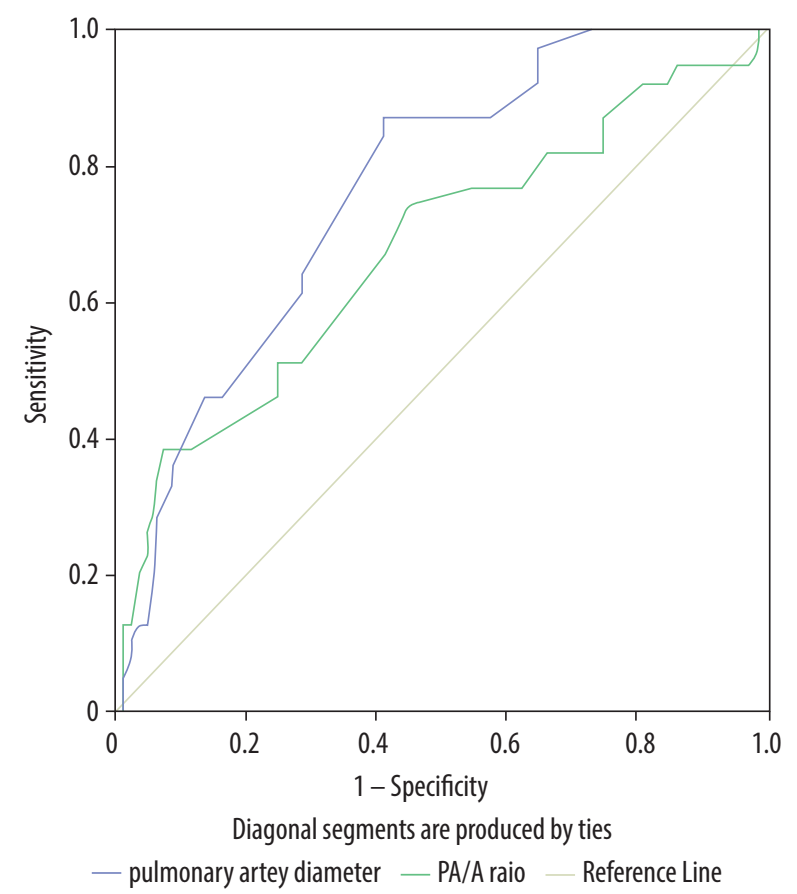

Figure 1. In patients below 18 , area under the ROC curve was 0.81 $(p=0.000)$ and $073(p=0.001)$ for PAD and PA/A ratio for identification of pulmonary hypertension, respectively. Using cut off of $19.25 \mathrm{rrma}$, the sensitivity and specificity of PAD for identification of PH was $82 \%$ and $70 \%$ respectively. At cut off $=0.94, \mathrm{PA} / \mathrm{A}$ ratio demonstrated the sensitivity $=83 \%$ and specificity $=55 \%$ 
Table 1. Baseline characteristics in patients with and without pulmonary hypertension

\begin{tabular}{|c|c|c|c|c|c|c|c|}
\hline Characteristics & & & $\begin{array}{l}\text { Overall } \\
(n=119)\end{array}$ & $\begin{array}{c}\text { PSAP } \\
<25 \mathrm{mmHg} \\
(n=39,32.8 \%)\end{array}$ & $\begin{array}{c}\text { PSAP } \\
\geq 25 \mathrm{mmHg} \\
(n=83,67.2 \%)\end{array}$ & $\begin{array}{c}\text { Pvalue } \\
\text { (univariate } \\
\text { analysis) }\end{array}$ & $\begin{array}{c}\text { Pvalue } \\
\text { (multiple } \\
\text { logistic } \\
\text { regression) }\end{array}$ \\
\hline \multirow[t]{14}{*}{ Demographics } & Age (yr) & & & & & & \\
\hline & & Overall & $14.86( \pm 6.4)$ & $13.85( \pm 7.08)$ & $16.94( \pm 4.04)$ & 0.003 & 0.97 \\
\hline & & Pediatrics & $11.36( \pm 4.68)$ & $10.14( \pm 4.74)$ & $14.26( \pm 2.78)$ & 0.000 & 0.3 \\
\hline & & Adults & $21.53( \pm 3.03)$ & $22( \pm 3.58)$ & $20.81( \pm 1.76)$ & 0.22 & \\
\hline & Gender & & & & & & \\
\hline & & Overall & & & & & \\
\hline & Male & & $74(62.2 \%)$ & $54(72.97 \%)$ & $20(27.02 \%)$ & 0.08 & \\
\hline & Female & & $45(37.8 \%)$ & $26(57.78 \%)$ & $19(42.22 \%)$ & & \\
\hline & & Pediatrics & & & & & \\
\hline & Male & & 49 (62.82\%) & $38(77.55 \%)$ & $11(22.45 \%)$ & 0.07 & \\
\hline & Female & & $29(31.18 \%)$ & $17(58.62 \%)$ & $12(41.39 \%)$ & & \\
\hline & & Adults & & & & 0.62 & \\
\hline & Male & & $25(60.97 \%)$ & $16(64 \%)$ & $9(36 \%)$ & & \\
\hline & Female & & $16(39.02 \%)$ & $7(43.75 \%)$ & $9(56.25 \%)$ & & \\
\hline \multirow[t]{10}{*}{ Anthropometrics } & $\mathrm{BMI}$ & & & & & & \\
\hline & & Overall & & & & & \\
\hline & $<20$ & & $106(84.1 \%)$ & $72(67.92 \%)$ & $34(32.08 \%)$ & 0.6 & \\
\hline & $20-25$ & & $13(10.4 \%)$ & $8(61.53 \%)$ & $5(38.46 \%)$ & & \\
\hline & & Pediatrics & & & & & \\
\hline & $<20$ & & 72 (92.3\%) & $51(70.8 \%)$ & $21(29.2 \%)$ & 0.8 & \\
\hline & $20-25$ & & $6(7.7 \%)$ & $4(66.7 \%)$ & $2(33.3 \%)$ & & \\
\hline & & Adults & & & & & \\
\hline & $<20$ & & $34(82.9 \%)$ & $21(61.8 \%)$ & $13(38.2 \%)$ & 0.8 & \\
\hline & $20-25$ & & $7(17.1 \%)$ & $4(57.1 \%)$ & $3(42.9 \%)$ & & \\
\hline \multirow[t]{12}{*}{ Arterial blood gas indices } & & Overall & & & & & \\
\hline & $\mathrm{pH}$ & & $7.39( \pm 0.06)$ & $7.4( \pm 0.06)$ & $7.39( \pm 0.06)$ & 0.89 & \\
\hline & $\mathrm{PCO}_{2}(\mathrm{mmHg})$ & & $45.37( \pm 11.01)$ & $44.11( \pm 11.7)$ & $47.99( \pm 9.03)$ & 0.011 & 0.37 \\
\hline & $\mathrm{HCO}_{3}(\mathrm{mmol} / \mathrm{l})$ & & $28.16( \pm 5.76)$ & $27.31( \pm 6.29)$ & $29.89( \pm 4.05)$ & 0.003 & 0.15 \\
\hline & & Pediatrics & & & & & \\
\hline & $\mathrm{pH}$ & & $7.39( \pm 0.057)$ & $7.4( \pm 0.06)$ & $7.34( \pm 0.05)$ & 0.66 & \\
\hline & $\mathrm{PCO}_{2}(\mathrm{mmHg})$ & & $44.18( \pm 11.64)$ & $43.5( \pm 11.76)$ & $45.83( \pm 11.43)$ & 0.03 & 0.64 \\
\hline & $\mathrm{HCO}_{3}(\mathrm{mmol} / \mathrm{l})$ & & $33.63( \pm 35.96)$ & $34.42( \pm 32.91)$ & $38.93( \pm 42.72)$ & 0.003 & 0.5 \\
\hline & & Adults & & & & & \\
\hline & $\mathrm{pH}$ & & $7.39( \pm 0.07)$ & $7.34( \pm 0.06)$ & $7.39( \pm 0.08)$ & 0.82 & \\
\hline & $\mathrm{PCO}_{2}(\mathrm{mmHg})$ & & $45.91( \pm 12.2)$ & $44.08( \pm 13.46)$ & $48.78( \pm 4.64)$ & 0.32 & \\
\hline & $\mathrm{HCO}_{3}(\mathrm{mmol} / \mathrm{l})$ & & $34.18( \pm 3.8)$ & $27.09( \pm 6.7)$ & $45.26( \pm 5.97)$ & 0.08 & \\
\hline \multirow[t]{9}{*}{ Pulmonary function test } & & Overall & & & & & \\
\hline & $\mathrm{FEV}_{1}$ & & $53.47( \pm 25.69)$ & $57.14( \pm 26.26)$ & $47.57( \pm 23.84)$ & 0.064 & \\
\hline & $\mathrm{FEV}_{1} / \mathrm{FVC}$ & & $97.12( \pm 14.73)$ & $94.14( \pm 15.98)$ & $98.79( \pm 12.33)$ & 0.61 & \\
\hline & & Pediatric & & & & & \\
\hline & $\mathrm{FEV}_{1}$ & & $56.91( \pm 28.23)$ & $55.28( \pm 31.86)$ & $46.3( \pm 26.11)$ & 0.29 & \\
\hline & $\mathrm{FEV}_{1} / \mathrm{FVC}$ & & $97.03( \pm 16.33)$ & $97.33( \pm 17.96)$ & $96.47( \pm 13.2)$ & 0.56 & \\
\hline & & Adults & & & & & \\
\hline & $\mathrm{FEV}_{1}$ & & $48.63( \pm 20.97)$ & $49.32( \pm 21.02)$ & $47.36( \pm 21.52)$ & 0.55 & \\
\hline & $\mathrm{FEV}_{1} / \mathrm{FVC}$ & & $97.26( \pm 11.95)$ & $94.16( \pm 12.00)$ & $102.12( \pm 10.44)$ & 0.04 & \\
\hline
\end{tabular}


Table 2. Pulmonary hemodynamic and vascular metrics in patients with and without pulmonary hypertension

\begin{tabular}{|c|c|c|c|c|c|c|}
\hline Characteristics & & $\begin{array}{c}\text { Overall } \\
(n=119)\end{array}$ & $\begin{array}{c}\text { PSAP }<25 \\
m m H g \\
(n=83,67.2 \%)\end{array}$ & $\begin{array}{c}\text { PSAP } \geq 25 \\
\text { mmHg } \\
(n=39,32.8 \%)\end{array}$ & $\begin{array}{l}\text { Pvalue } \\
\text { (univariate } \\
\text { analysis) }\end{array}$ & $\begin{array}{c}P \text { value } \\
\text { (multiple logistic } \\
\text { regression) }\end{array}$ \\
\hline \multicolumn{7}{|c|}{ Pulmonary systolic pressure (mmHg) } \\
\hline & Overall & $24.39( \pm 8.85)$ & $20.05( \pm 7.93)$ & $33.30( \pm 10.98)$ & 0.00 & \\
\hline & Pediatrics & $24.08( \pm 8.46)$ & $19.94( \pm 7.3)$ & $34( \pm 10.69)$ & 0.00 & \\
\hline & Adults & $24.97( \pm 9.32)$ & $20.28( \pm 8.9)$ & $32.31( \pm 11.66)$ & 0.000 & \\
\hline \multicolumn{7}{|c|}{ Pulmonary artery diameter (mm) } \\
\hline & Overall & $19.88( \pm 4.91)$ & $18.43( \pm 4.72)$ & $22.86( \pm 3.86)$ & 0.000 & 0.005 \\
\hline & Pediatrics & $18.73( \pm 5.13)$ & $17.10( \pm 4.64)$ & $22.63( \pm 4.4)$ & 0.000 & 0.03 \\
\hline & Adults & $22.06( \pm 3.61)$ & $21.34( \pm 3.49)$ & $23.19( \pm 3.60)$ & 0.7 & \\
\hline \multicolumn{7}{|l|}{$\mathrm{PA} / \mathrm{A}$} \\
\hline & Overall & $0.98( \pm 0.12)$ & $0.95( \pm 0.11)$ & $1.03( \pm 0.13)$ & 0.002 & 0.79 \\
\hline & Pediatrics & $0.99( \pm 0.12)$ & $0.97( \pm 0.11)$ & $1.05( \pm 0.13)$ & 0.001 & 0.77 \\
\hline & Adults & $0.96( \pm 0.13)$ & $0.94( \pm 0.12)$ & $1.00( \pm 0.14)$ & 0.125 & \\
\hline
\end{tabular}

specificity of PAD for identification of $\mathrm{PH}$ were $82 \%$ and $70 \%$, respectively. At a cut-off value of 0.94 , the PA/A ratio demonstrated a sensitivity of $83 \%$ and specificity of $55 \%$. The results were similar for total CF and cohort and paediatric CF patients.

Variables with a significant correlation with PSAP (age, $\mathrm{PCo}_{2}, \mathrm{HCo}_{3}, \mathrm{PAD}, \mathrm{PA} / \mathrm{A}$ ratio) based on univariate analysis were further analysed by multiple regression test, which revealed PAD as the only independent predictor of $\mathrm{PH}$ in total CF patients $(p=0.006)$ and in paediatric CF patients $(p=0.033)$. In adults groups, no independent relationship was found between pulmonary artery hypertension and CT-measured vascular metrics.

\section{Discussion}

The current study provided new insight into the age-dependent performance of CT-measured PAD to predict $\mathrm{PH}$ in CF patients. PAD may serve as a reliable tool to identify $\mathrm{PH}$ in paediatric patients with CF; however, no significant association was found between the level of PASP and pulmonary artery diameter in adults.

Few studies are available investigating various aspects of $\mathrm{PH}$ in $\mathrm{CF}$. It has been shown that $\mathrm{PH}$ is frequently seen in advanced CF (57\%) [10]. In the present study, the frequency of $\mathrm{PH}$ was found to be $32.8 \%$. This difference may be at least partly due to the lower prevalence of advanced CF in the current study (2.5\%).

Pulmonary hypertension is a pathophysiological condition caused by a variety of diseases mediated via different underlying anatomical and physiological mechanisms. In chronic obstructive pulmonary disease (COPD), recent studies have demonstrated that a direct toxic effect of tobacco on the intima proceed vascular remodelling via abnormal production of the mediators controlling vasodilation and vasoconstriction and also abnormal intimal cell proliferation $[11,12]$; however, in CF patients, vascular remodelling is strongly influenced by the alveolar hypoxia as well as a chronic systemic inflammatory condition $[13,14]$. Augmentation index (AIx), as a significant predictor of arterial stiffness with a potential association with systemic inflammation, has been demonstrated to have higher values in the adult CF population, indicating an accelerated vascular ageing process in this cohort [14]. Despite an independent relationship in paediatric $\mathrm{CF}$, the present study failed to demonstrate any striking correlation between pulmonary artery diameter and $\mathrm{PH}$ in adult CF. Taking this into consideration, one possible explanation is the well-established premature vascular aging and advanced arterial stiffness in adult CF compared to the paediatric group, preventing a linear relationship between pulmonary artery pressure and pulmonary artery diameter.

Most researches demonstrated that pulmonary haemodynamic indices are independent of lung function status in CF patients [13], which is in accordance with the results obtained in the present study. Such results provided evidence for the major limitation of PFT to be solely applied for disease monitoring in CF patients, and they support the potential value of CT-measured thoracic vascular diameter as a complementary test to PFT for comprehensive disease monitoring in paediatric CF patients.

Several reports revealed a significant correlation between obesity and PH. One study demonstrated that $72 \%$ of subjects with BMI $>30 \mathrm{~kg} / \mathrm{m}^{2}$ showed haemodynamic changes in pulmonary circulation, including venous hypertension and alveolar capillary haemangiomatosis, indicating of an underlying PH [15]. Another study demonstrated that the frequency of obesity was significantly higher in patients with $\mathrm{PH}$ than in those with normal pul- 
monary pressure [16]. The same result was also observed in COPD patients with pulmonary hypertension - mean BMI was significantly higher in patients with $\mathrm{PH}$ (28 vs. 25, $p=0.04$ ) [17]. However, the current study failed to provide any association between BMI and pulmonary systolic arterial pressure as well as CT vascular metrics. This discrepancy could be explained by the confounding effect of underlying malnutrition status most frequently seen in CF patients as a result of pulmonary function disturbance and gastrointestinal complications including pancreatic insufficiency, steatorrhoea, hepatic steatosis, and biliary fibrosis.

The present study has some major drawbacks. Retrospective data collection from a single CF centre may limit the validity of results to the CF patient population. However, it should be noted that Masih Daneshvari Hospital, as a tertiary referral centre for CF with a comprehensive $\mathrm{CF}$ registry, provides a wide coverage of $\mathrm{CF}$ patients in Iran. The impact of CT vascular metrics to predict $\mathrm{PH}$ on clinical outcome needs to be further investigated. None of the patients were candidates for right-sided heart catheterisation, and hence the exact value of haemodynamic parameters measured by gold standard were not available; however, the current results may be less influenced by the selection bias in the studies performed on RVH candidate cohorts. Associated pulmonary parenchymal involvement as a major determinant of survival with a potential impact on pulmonary arterial systolic pressure was not evaluated in this study. Small subsets of patients in age groups and limited age range of adult CF (up to 33 years old) should be considered as the important consideration for the present results.

In conclusion, the current study provided evidence for the age-dependent performance of CT-based pulmonary artery diameter, as an easily obtainable measurement, to predict $\mathrm{PH}$. PAD correlates well with $\mathrm{PH}$ in paediatric patients with CF, and this relationship is independent of age, lung function test, and arterial blood gas indices. In this regard, CT-measured thoracic vascular diameter may be recommended as a complementary test to pulmonary function test for monitoring of disease progression in childhood CF. However, the added value of CT-based follow-up approach in paediatric CF should be further validated by large-scale prospective survival studies considering excess radiation exposure.

\section{Conflict of interest}

The authors report no conflict of interest.

\section{References}

1. Hayes Jr D, Tobias JD, Mansour HM, et al. Pulmonary hypertension in cystic fibrosis with advanced lung disease. Am J Respir Crit Care Med 2014; 190: 898-905.

2. Arcasoy SM, Christie JD, Ferrari VA, et al. Echocardiographic assessment of pulmonary hypertension in patients with advanced lung disease. Am J Respir Crit Care Med 2003; 167: 735-740.

3. Fisher MR, Forfia PR, Chamera E, et al. Accuracy of Doppler echocardiography in the hemodynamic assessment of pulmonary hypertension. Am J Respir Crit Care Med 2009; 179: 615-621.

4. Helbich TH, Heinz-Peer G, Eichler I, et al. Cystic fibrosis: CT assessment of lung involvement in children and adults. Radiology 1999; 213: $537-544$

5. Fraser KL, Tullis DE, Sasson Z, et al. Pulmonary hypertension and cardiac function in adult cystic fibrosis: role of hypoxemia. Chest 1999; 115: 1321-1328.

6. McLaughlin VV, Presberg KW, Doyle RL, et al. Prognosis of pulmonary arterial hypertension: ACCP evidence-based clinical practice guidelines. Chest 2004; 126 (1 Suppl): 78S-92S.

7. Ussavarungsi K, Whitlock JP, Lundy TA, et al. The significance of pulmonary artery size in pulmonary hypertension. Diseases 2014; 2: 243-259.

8. Akay HO, Ozmen CA, Bayrak AH, et al. Diameters of normal thoracic vascular structures in pediatric patients. Surg Radiol Anat 2009; 31: 801-807.

9. Compton GL, Florence J, MacDonald C, et al. Main pulmonary artery-to-ascending aorta diameter ratio in healthy children on MDCT. Am J Roentgenol 2015; 205: 1322-1325.
10. Hayes D, Higgins RS, Kirkby S, et al. Impact of pulmonary hypertension on survival in patients with cystic fibrosis undergoing lung transplantation: an analysis of the UNOS registry. J Cyst Fibros 2014; 13: 416-423.

11. Wright JL, Levy RD, Churg A. Pulmonary hypertension in chronic obstructive pulmonary disease: current theories of pathogenesis and their implications for treatment. Thorax 2005; 60: 605-609.

12. Zakynthinos E, Daniil Z, Papanikolaou J, Makris D. Pulmonary hypertension in COPD: pathophysiology and therapeutic targets. Curr Drug Targets 2011; 12: 501-513.

13. Fraser KL, Tullis DE, Sasson Z, et al. Pulmonary hypertension and cardiac function in adult cystic fibrosis: role of hypoxemia. Chest 1999; 115: 1321-1328.

14. Hull JH, Garrod R, Ho TB, et al. Increased augmentation index in patients with cystic fibrosis. Eur Respir J 2009; 34: 1322-1328.

15. Haque AK, Gadre S, Taylor J, et al. Pulmonary and cardiovascular complications of obesity: an autopsy study of 76 obese subjects. Arch Pathol Lab Med 2008; 132: 1397-1404.

16. Chan AL, Juarez MM, Shelton DK, et al. Novel computed tomographic chest metrics to detect pulmonary hypertension. BMC Med Imaging 2011; $11: 7$.

17. Shin S, King CS, Brown AW, et al. Pulmonary artery size as a predictor of pulmonary hypertension and outcomes in patients with chronic obstructive pulmonary disease. Respir Med 2014; 108 : 1626-1632. 